\title{
OLANZAPINE INDUCED HYPONATREMIA AND RHABDOMYOLYSIS
}

\author{
Younes N'joumi ${ }^{1}$, Haimeur $\mathrm{Y}^{1}$, Harfaoui $\mathrm{Y}^{1}$, Benhlima $\mathrm{A}^{1}$, Elhamzaoui $\mathrm{H}^{1}$, and Alilou $\mathrm{M}^{1}$ \\ ${ }^{1}$ Universite Mohammed V de Rabat Faculte de Medecine et de Pharmacie de Rabat
}

November 21, 2021

\begin{abstract}
Rapid-onset hyponatremia as well as rhabdomyolysis are rare, but potential, complications of olanzapine treatment. We report a case of sudden-onset hyponatremia associated to a sever rhabdomyolysis resulting in a coma necessitating intensive care unit admission. His evolution was favorable after correction of all his metabolic disorders and Olanzapine suspension.
\end{abstract}

\section{OLANZAPINE INDUCED HYPONATREMIA AND RHABDOMYOLYSIS}

$\mathrm{N}^{\prime}$ joumi $\mathrm{Y}^{1^{*}}$, Haimeur $\mathrm{Y}^{1^{*}}$, Harfaoui $\mathrm{Y}^{1^{*}}$, Benhlima A ${ }^{1^{*}}$, Elhamzaoui $\mathrm{H}^{1^{*}}$, Alilou $\mathrm{M}^{1^{*}}$

1 Emergency Department, IBN SINA Hospital / Faculty of Medicine and Pharmacy (University Mohammed V), Rabat, Morocco.

Corresponding author: N'joumi Younes :younes.njoumi@um5r.ac.ma

N'joumi Younes :younes.njoumi@um5r.ac.ma

Haimeur Yassine : yassine.haimeur1@gmail.com

El Harfaoui Yassine : yassineelharfaoui@gmail.com

Benhlima Abdelkader : Abdel.benhlima@gmail.com

Elhamzaoui Hamza : elhamzaouihamza6@gmail.com

Alilou Mustapha : aliloumust@yahoo.fr

N.B: Written informed consent was obtained from the patient to publish this report in accordance with the journal's patient consent policy.

\section{ABSTRACT}

Rapid-onset hyponatremia as well as rhabdomyolysis are rare, but potential, complications of olanzapine treatment. Hyponatremia, secondary to atypical antipsychotic use, has been reported in many case reports and is thought to be associated with an inappropriate anti-diuretic hormone secretion syndrome (ISADH). We report a case of sudden-onset hyponatremia associated to a sever rhabdomyolysis resulting in a coma necessitating intensive care unit admission. His evolution was favorable after correction of all his metabolic disorders and Olanzapine suspension.

\section{KEY CLINICAL MESSAGE}

Disturbances of consciousness are very common in emergency departments, and metabolic causes are often at the root of them. In the case of dysnatremia, drug-related causes should always be considered. 
KEYWORDS: Olanzapine; Hyponatremia; Rhabdomyolysis; Side effects INTRODUCTION

The association between hyponatremia and antipsychotic drug use has been widely reported in the literature, however, it remains poorly understood and underestimated by practitioners, as does rhabdomyolysis.

We report a patient with both symptomatic profound hyponatraemia and rhabdomyolysis following the use of Olanzapine.

\section{CASE REPORT}

Mr M.R, 57 years-old, was admitted to the intensive care unit of the emergency room for apyretic loss of consciousness. He had a history of schizophrenic disorders for which he was treated with Olanzapine $10 \mathrm{mg} /$ day. His family reported that the symptoms were poorly controlled pushing the patient to increase the daily dosage of his medication by himself.

The day of admission, the patient was found unconscious by his family, prompting a visit to the emergency room.

On admission, his Glasgow consciousness score was 6, pupils were equal and reactive, with no signs of focalization or extrapyramidal syndrome and no signs of seizures.

He was polypneic at $34 \mathrm{cpm}$, his saturation was $80 \%$ on room air with paradoxical breathing and respiratory pauses. Pulmonary auscultation was unremarkable.

Hemodynamically, BP was 115/70 mmHg, HR $110 \mathrm{bpm}$, RT < 3sec. Capillary glycemia was 2g/l, and the temperature $37.3^{\circ}$. His diuresis was preserved and urine was slightly concentrated.

After conditioning, the patient was intubated and then sedated, with a cerebral CT scan that came back normal, and a biological workup that came back in favour of hyponatremia at $106 \mathrm{mEq} / \mathrm{l}$ and rhabdomyolysis with CPK at $19829 \mathrm{UI} / \mathrm{l}, \mathrm{LDH}$ at $2254 \mathrm{UI} / \mathrm{l}$ and concomitant hyperkaliemia at $6.9 \mathrm{meq} / \mathrm{l}$.

Management consisted of correction of the natremia and forced alkaline diuresis.

The rest of the work-up showed a urinary osmolarity of $638 \mathrm{mOsm}$ with a natriuresis of $190 \mathrm{mEq} / \mathrm{l}$ and a blood osmolarity of $219 \mathrm{mOsm}$ and thus met all the major criteria for inappropriate secretion of anti-diuretic hormone (ISADH). The etiological investigation came back negative, and the patient was diagnosed with ISADH with rhabdomyolysis secondary to Olanzapine.

The patient was subsequently transferred to a medical intensive care unit for further management. The evolution was marked by a complete awakening of the patient, extubation on day 6 of his admission, after correction of the natremia $(146 \mathrm{mEq} / \mathrm{l})$, decrease in the markers of rhabdomyolysis from day 3 (CPK: 1681 UI/l, LDH: $503 \mathrm{UI} / \mathrm{l}$ ) and normal kaliemia.

\section{DISCUSSION}

Hyponatremia is the direct consequence of inappropriate ADH secretion. Depending on its depth, the clinical picture may range from asymptomatic patient to coma or cardiorespiratory arrest, via neurological disorders such as convulsions, stupor, agitation and confusion if it is acute, or apathy, anorexia, muscle cramps and memory and balance disorders when it is progressive. The clinical picture thus depends mainly on the speed of onset and depth of hyponatremia [1].

ISADH combines several symptoms first described by Schwartz and Bartter in 1967, resulting in normovolemic hyponatremia [2]. However, it remains a diagnosis of elimination in the presence of hyponatremia. The diagnostic criteria are presented in Table 1 [1].

Table 1 : diagnostic criteria for ISADH [1] 


\section{Major criteria}

Plasma osmolarity $<280 \mathrm{mOsm} / \mathrm{L}$ Inappropriate urine osmolarity $>150 \mathrm{mOsm} / \mathrm{L}$ Normal extracellular volume: no orthost

The most common causes of ISADH are pulmonary, neurological or paraneoplastic [1]. However, a drugrelated cause should always be sought. The drugs most frequently associated with ISADH are shown on Table $2[1]$.

Table 2: Drugs that increase risks of ISADH syndrome [1]

Antidepressants: tricyclics, serotonin reuptake inhibitors, IMAOS Anti-psychotics: phenotiazine, haloperidol Anti-epileptics: carbamazepine, valproic acid Anti-cancer drugs: alacaloids, platinum salts, alkylating agents, methotrexate, Interferon, monoclonal antibodies Opioid analgesics: tramadol, morphine Miscellaneous:

Drugs that increase the production of ADH by the hypothalamus

Medication that potentiates the effect of ADH proton pump inhibitors, nicotine, "ecstasy" (MDMA), clofibrate

Anti-epileptics: carbamazepine, lamotrigine Anti-diabetics: chlorpropamide, tolbutamide Anti-cancer drugs: intravenous cyclophosphamide Non-steroidal anti-inflammatory drugs Desmopressin, oxytocin, vasopressin

Olanzapine is a new atypical antipsychotic drug with proven efficacy in several psychiatric conditions such as schizophrenia and autism. In a pharmacovigilance study of Olanzapine in 8858 patients in the UK, the main adverse effects were: somnolence with sedative effect, extra-pyramidal signs, weight gain, lassitude, agitation, liver abnormalities... [3]

In a study based on cases reported to the WHO International Drug Monitoring Collaboration, Mannesse and all found that Olanzapine was the second most common atypical antipsychotic associated with hyponatremia/ISADH, after risperidone [4].

In animal models, it has been shown that Dopamine has an inhibitory effect on ADH secretion. This effect can be blocked by dopamine receptor antagonists. Olanzapine is a selective monoaminergic antagonist with a high affinity for dopamine receptors and causes ISADH through its antagonism to them $[5,6]$.

The association between Olanzapine use and rhabdomyolysis and CPK elevation has also been reported in the literature [7-9]. The exact mechanism associating them remains to be elucidated, however some authors suggest the existence of an important role for serotonin (5-HT), as Olanzapine would have a more potent activity than antagonists of the serotonin receptors of Dopamine. 5-HT is believed to be toxic to skeletal muscle, which contains high-affinity receptors in the sarcolemma, and the cell membrane $[10,11]$.

In our patient, the elevation of $\mathrm{CPK}$ and LDH associated with the existence of hyperkaliemia made it possible to retain the diagnosis of rhabdomyolysis, the search for myoglobinuria was not performed. The questioning and clinical examination did not reveal any signs of muscle lysis other than toxic, thus allowing Olanzapine to be considered the direct cause.

The chronology of the symptoms that appeared and worsened concomitantly with the untimely intake of Olanzapine, as well as the improvement and correction of the hyponatremia and rhabdomyolysis following the cessation of the said drug and therapeutic measures, without recurrence, support our diagnostic hypothesis. 


\section{CONCLUSION}

The association between use of Olanzapine and occurrence of episodes of hyponatremia has been reported quite frequently in the literature, as has rhabdomyolysis. They do not seem to be correlated with any risk factor or toxic dose and their occurrence is sporadic. Similarly, the concomitant occurrence of these two complications in the same patient remains exceptional.

Their diagnosis remains of elimination, but this does not delay treatment which is mostly symptomatic.

\section{AUTHORS CONTRIBUTION:}

N'joumi Younes : is the main author of the manuscript

Haimeur Yassine : has been involved in drafting in the manuscript

El Harfaoui Yassine : has been involved in drafting in the manuscript

Benhlima Abdelkader : has been involved in drafting in the manuscript

Elhamzaoui Hamza: has revised the manuscript

Alilou Mustapha: has given final approval of the version to be published

\section{BIBLIOGRAPHIE}

[1] Passeron A, Dupeux S, Blanchard A. Hyponatrémie : de la physiopathologie a la pratique. Rev Medecine Interne 2010;31:277-86. https://doi.org/10.1016/j.revmed.2009.03.369.

[2] Bart'Ter C, Schwartz B. The Syndrome of Inappropriate Secretion of Antidiuretic Hormone n.d.:17.

[3] Biswas PN, Wilton LV, Pearce GL, Freemantle S, Shakir SAW. The pharmacovigilance of olanzapine: results of a post-marketing surveillance study on 8858 patients in England. J Psychopharmacol (Oxf) 2001;15:265-71. https://doi.org/10.1177/026988110101500405.

[4] Mannesse CK, van Puijenbroek EP, Jansen PAF, van Marum RJ, Souverein PC, Egberts TCG. Hyponatraemia as an Adverse Drug Reaction of Antipsychotic Drugs: A Case-Control Study in VigiBase. Drug Saf 2010;33:569-78. https://doi.org/10.2165/11532560-000000000-00000.

[5] Wells T, Forsling M. Aminergic control of vasopressin secretion in the conscious rat. J Physiol Pharmacol 1992;43:57-62.

[6] Yamaguchi K. Inhibitory role of periventricular dopaminergic mechanisms in hemorrhage-induced vasopressin secretion in conscious rats n.d.:4.

[7] Rosebraugh CJ, Flockhart DA, Yasuda SU, Woosley RL. Olanzapine-Induced Rhabdomyolysis. Case Rep n.d.:4.

[8] Karakaya P, Yiş U, Kurul SH, Türkmen MA. Rhabdomyolysis Associated With Olanzapine Treatment in a Child With Autism: Pediatr Emerg Care 2010;26:41-2. https://doi.org/10.1097/PEC.0b013e3181c39a22.

[9] Ribeyron S, Guy C, Koenig M, Cathébras P. Rhabdomyolyse et élévation des enzymes musculaires sous olanzapine. Rev Médecine Interne 2009;30:477-85. https://doi.org/10.1016/j.revmed.2008.12.024.

[10] Meltzer HY. Massive serum creatine kinase increases with atypical antipsychotic drugs: what is the mechanism and the message? Psychopharmacology (Berl) 2000;150:349-50. https://doi.org/10.1007/s002130000465.

[11] Meltzer HY. Skeletal muscle necrosis following membrane-active drugs plus serotonin. J Neurol Sci 1976;28:41-56. https://doi.org/10.1016/0022-510X(76)90046-0.

\section{CONFLICT OF INTEREST:}

The authors declare no competing interest. 


\section{DATA AVAILABILITY:}

The data that support the findings of this study are available from the corresponding author upon reasonable request. 